\title{
ÉTUDE EXPÉRIMENTALE DU CYCLE BIOLOGIQUE DE ECHINOSTOMA TOGOENSIS N. SP., PARASITE A L'ÉTAT LARVAIRE DE BIOMPHALARIA PFEIFFERI AU TOGO.
}

\author{
J. JOURDANE* et S. D. KULO**
}

RÉSUMÉ. L'adulte et les stades larvaires de Echinostoma togoensis sont décrits. E. togoensis évolue au stade rédie dans la glande génitale de Biomphalaria pfeifferi qu'il stérilise totalement. Les métacercaires se développent soit dans la cavité péricardique de divers Pulmonés aquatiques, soit dans le rein des têtards d'Amphibiens. L'adulte a été obtenu expérimentalement exclusivement chez la souris blanche.

\section{Experimental study of the life cycle of Echinostoma togoensis n. sp., a larval parasite of Biomphalaria pfeifferi in Togo.}

SUMMARY. The adult and larval stages of Echinostoma togoensis are described. E. togoensis develops into rediae in the ovotestis of Biomphalaria pfeifferi which is totally sterilized. The metacercariae were recovered either in the pericardial cavity of several aquatic Pulmonata, or in the kidney of amphibian tadpoles. The adult was experimentally obtained in the laboratory mice exclusively.

\section{Introduction}

Une mission de prospection effectuée au Togo en mai 1978, dans le cadre de recherches sur l'écologie de la transmission de la bilharziose intestinale, nous a permis de recenser chez Biomphalaria pfeifferi, outre les stades larvaires de Schistosoma mansoni, ceux de plusieurs autres Digènes parasites. Dans une station du Nord Togo (Lama-Kara), de nombreux Planorbes hébergeaient les formes larvaires d'un échinostome à partir desquelles nous avons pu reconstituer expérimentalement le cycle évolutif du parasite. Cedex.

* Département de Biologie animale (Directeur: Pr. C. Combes), Université, F 66025 Perpignan

** Laboratoire de Zoologie, École des Sciences, BP 1515 Lome (Togo).

Accepté le I5 janvier I98r. 
Dans l'exposé qui suit, nous nous proposons de décrire les modalités de l'évolution de cet échinostome et ses différents stades évolutifs. Cette espèce n'ayant pu être identifiée aux échinostomes connus d'Afrique, nous avons été conduits à la considérer comme nouvelle.

\section{Schéma du cycle}

D'après nos résultats expérimentaux, le cycle biologique d'Echinostoma togoensis se déroule selon le schéma suivant :

- L'œuf, éliminé avec les fèces dans l'eau, éclôt à partir du $12^{\mathrm{e}}$ jour à une température voisine de $+26^{\circ} \mathrm{C}$;

- le miracidium, cilié, nageant, pénètre activement chez des Planorbidae du genre Biomphalaria où il évolue en sporocyste ; le développement intramolluscal comprend au moins deux générations de rédies parasistant préférentiellement la glande génitale du Mollusque ;

- les cercaires, du type échinostome, produites par les rédies, quittent le Mollusque et pénètrent activement soit chez un deuxième Mollusque, soit chez des têtards d'Amphibiens ;

- l'hôte définitif s'infeste en ingérant les hôtes intermédiaires parasités par les métacercaires.

\section{Description des stades évolutifs}

\section{1) Miracidium (fig. 1b.)}

Le miracidium, de forme tronconique, mesure 105-150 $\mu \mathrm{m}$ sur 60-75 $\mu \mathrm{m}$ (moyenne : 125 sur $70 \mu \mathrm{m}$ ). Il est pourvu d'une tache oculaire en forme de X située dans la région antérieure. Les cellules épidermiques ciliées sont disposées sur quatre étages selon la séquence : $6-6-4-2$.

Le système excréteur se compose de deux protonéphridies dont les orifices externes s'ouvrent ventralement et dorsalement entre le troisième et le quatrième étage des cellules épithéliales.

L'imprégnation au nitrate d'argent des miracidiums permet de mettre en évidence de façon constante des éléments argyrophiles très caractéristiques : au niveau du térébratorium ( $f i g .1 c$ ), on note la présence de six éléments groupés 3 par 3 ; au niveau des cellules de l'étage apical se trouvent six structures logées chacune dans une légère sinuosité du bord inférieur de la cellule.

\section{2) Sporocyste (fig. 1a)}

Les sporocystes parasitent le ventricule et l'aorte du Mollusque, la localisation ventriculaire étant la plus fréquente (70\% des cas). Dans les fortes infestations 
(A)

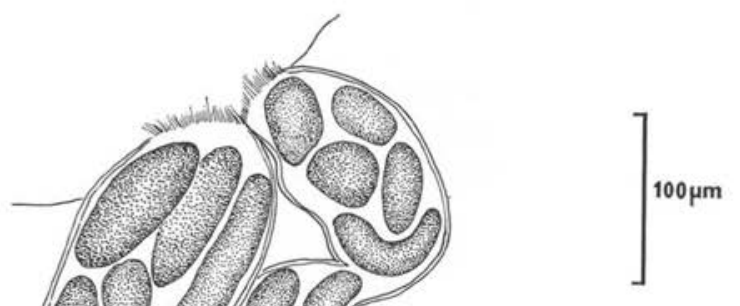

(B)
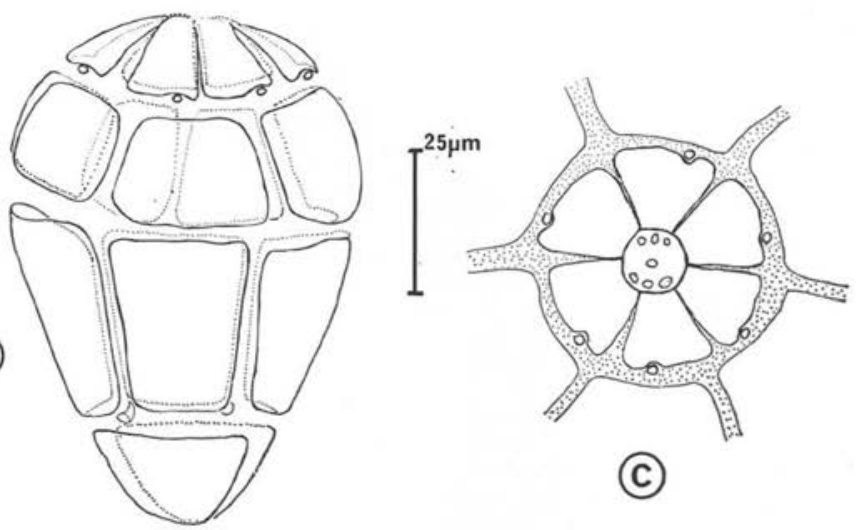

(C)

FIG. I. - Echinostoma togoensis n. sp. A : Sporocystes renfermant des balles germinales et des rédies mères. $\mathrm{B}$ : Miracidium, disposition des cellules ciliées. $\mathrm{C}:$ Miracidium : structures argyrophiles du terebratoium (vue apicale).

(à partir de 10 miracidiums), des sporocystes peuvent être exceptionnellement rencontrés hors du cœur, dans la cavité péricardique, la cavité pulmonaire ou au voisinage immédiat de l'aorte. Le sporocyste se présente sous la forme d'un sac allongé mesurant à 12 jours 250-600 $\mu \mathrm{m}$ sur 110-200 $\mu \mathrm{m}$ (moyenne : 500 sur $150 \mu \mathrm{m}$ ). Il adhère très fortement par une extrémité au myocarde du Mollusque; l'autre extrémité, libre, bat dans l'hémolymphe au rythme des pulsations cardiaques. Chez les sporocystes ayant atteint leur développement optimum, 2 à 4 rédies-mères sont visibles parmi des balles germinatives.

Après 18 jours, le sporocyste apparaît sous une teinte jaune orangé qui marque le début de sa phase de dégénérescence. 
3) Rédie-mère (fig. 2a)

Les rédies-mères sont libérées dans les tissus du Mollusque à partir du $10^{\mathrm{e}}$ jour. Une fraction importante d'entre elles poursuit son développement dans le cœur et les vaisseaux efférents avant d'arriver dans la glande génitale du Planorbe. Les rédies-

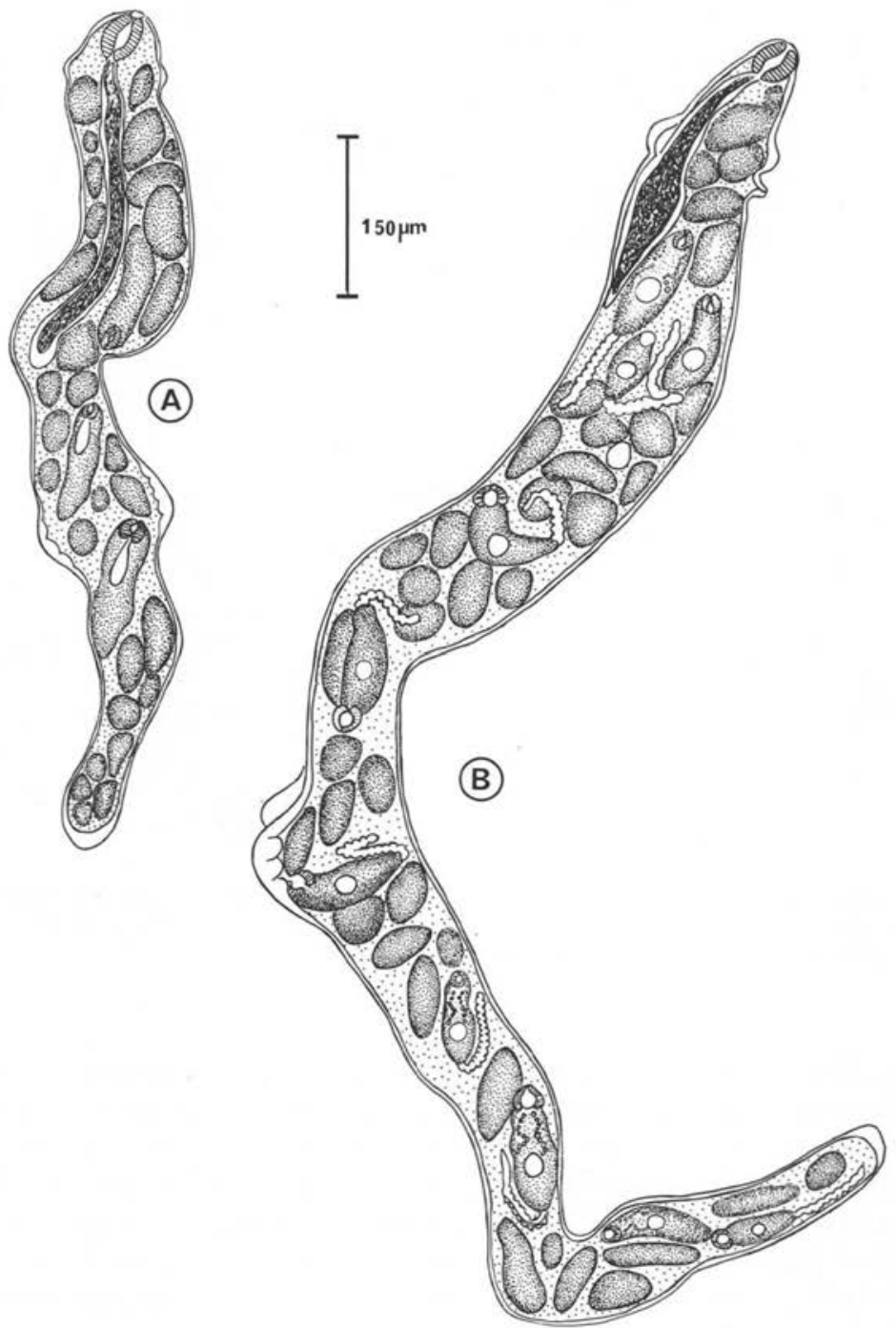

FIG. 2. - Echinostoma togoensis n. sp. A : Rédie mère. B : Rédie fille. 
mères sont visibles dans la région génitale environ vers le $13 \mathrm{e}$ jour après l'infestation ; ces dernières mesurent 700-1 $500 \mu \mathrm{m}$ de longueur sur 150-250 $\mu \mathrm{m}$ de largeur; elles présentent un collier musculeux bien marqué, parapharyngien, et des appendices locomoteurs situés dans la région antérieure du tiers postérieur du corps. Le système digestif comprend un pharynx de $50 \mu \mathrm{m}$ de diamètre environ et un caecum long atteignant presque la moitié du corps.

\section{4) Rédie-fille (fig. 2b)}

Dès le $13^{e}$ jour, les rédies-filles sont rencontrées dans la glande génitale. A partir de ce stade, les tissus viscéraux et principalement la glande génitale vont être progresivement envahis par les larves du parasite. A leur développement optimum, lesrédies-filles mesurent $1050-3050 \mu \mathrm{m}$ sur $150-320 \mu \mathrm{m}$; leur tégument est jauneorangé. Les déplacements des rédies sont permis grâce au collier musculeux, à deux fourchons ventraux et à une puissante musculature longitudinale. Postérieurement au collier et en position dorsale se trouve le pore de sortie des cercaires. Le diamètre du pharynx varie de 35 à $60 \mu \mathrm{m}$. Chez les rédies-filles, le caecum digestif est relativement moins développé que chez les rédies-mères : sa longueur est en moyenne le double de la distance extrémité antérieure-collier.

\section{5) Cercaire (fig. 3a)}

La cercaire est du type Échinostome. Le corps cercarien est de forme ovale. A son extrémité antérieure, un collier céphalique porte 37 épines de $12 \mu \mathrm{m}$ de longueur en moyenne. La ventouse orale occupe une position subterminale; la ventouse ventrale, plus importante, est située dans la moitié postérieure du corps. La queue contractile, présente une memprane ondulante ventrale unique, deux membranes ondulantes dorsales bien séparées et deux membranes ondulantes latérales très fines. L'insertion des membranes sur la queue est conforme au schéma de la figure $3 c$.

Les dimensions de la cercaire rapportées ci-dessous correspondent à celles de 30 larves vivantes mesurées entre lame et lamelle sans aplatissement (les chiffres entre parenthèse représentent les moyennes).

Longueur du corps

Largeur du corps

Longueur de la queue

Diamètre de la ventouse orale

Diamètre de la ventouse ventrale

Pharynx

$$
\begin{aligned}
& 190-265 \mu \mathrm{m}(245) \\
& 160-200 \mu \mathrm{m}(180) \\
& 365-470 \mu \mathrm{m}(390) \\
& 40 \text { à } 60 \mu \mathrm{m}(50) \\
& 46 \text { à } 70 \mu \mathrm{m}(60) \\
& 20-45 \text { sur } 16-20 \mu \mathrm{m}(30 \times 18)
\end{aligned}
$$

L'appareil digestif comprend un prépharynx bien individualisé, un pharynx, un oesophage et deux caecums pleins s'étendant jusqu'à la vessie. Nous n'avons pu mettre en évidence en contraste interférentiel, ni après coloration au rouge neutre, de glandes para-oesophagiennes.

Le système excréteur est du type sténostome. Il se compose de 18 paires de protonéphridies groupées en triplets. Les troncs collecteurs primaires descendants ren- 

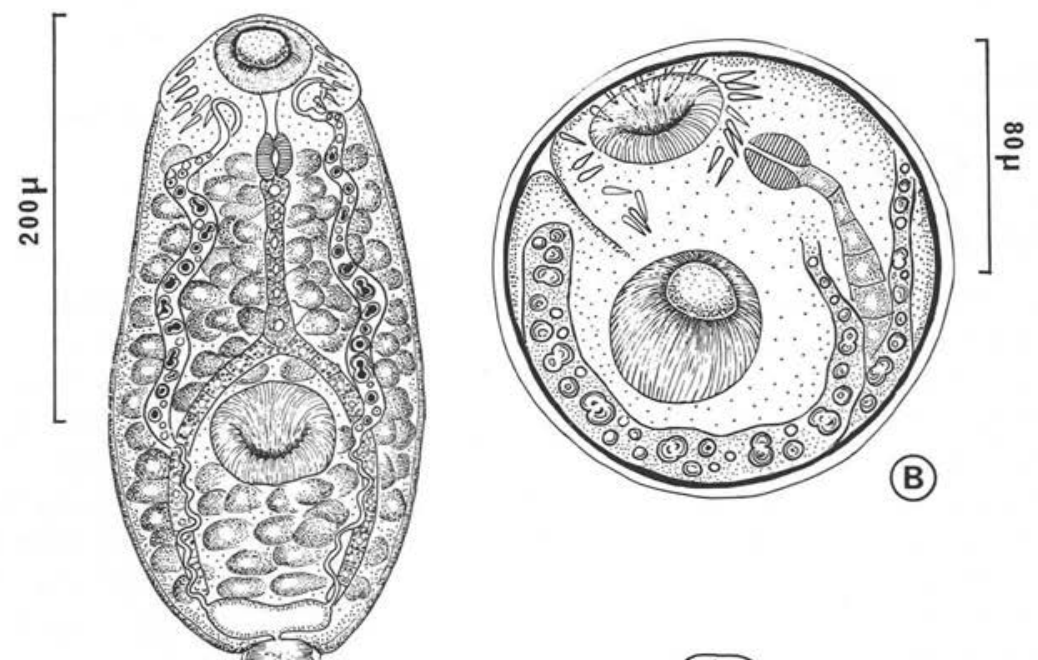

(A)
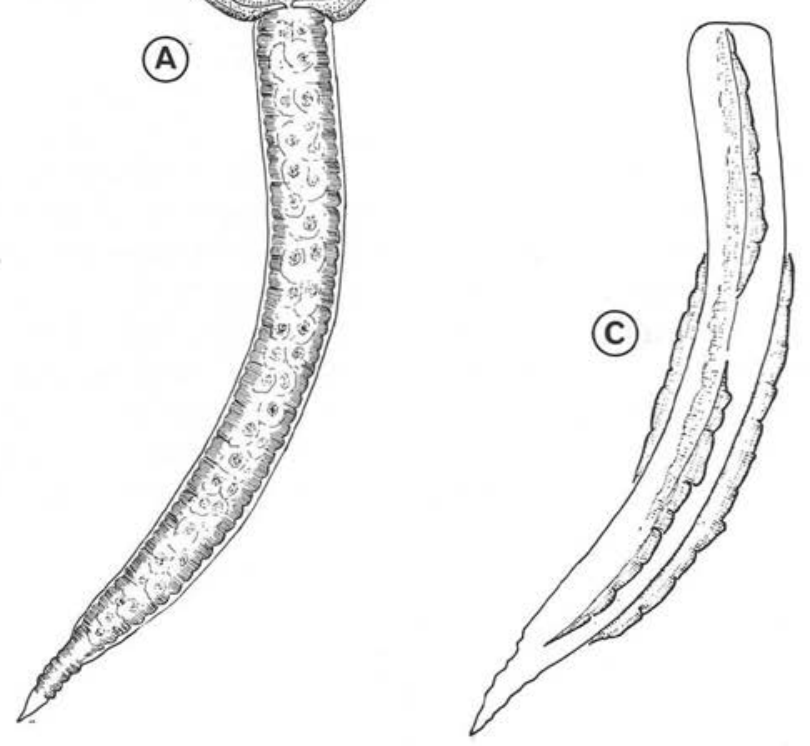

FIG. 3. - Echinostoma togoensis n. sp. A : Cercaire, morphologie générale (vue ventrale).B:Métacercaire enkystée. C : Cercaire, disposition des membranes ondulantes sur la queue (vue ventrale). 

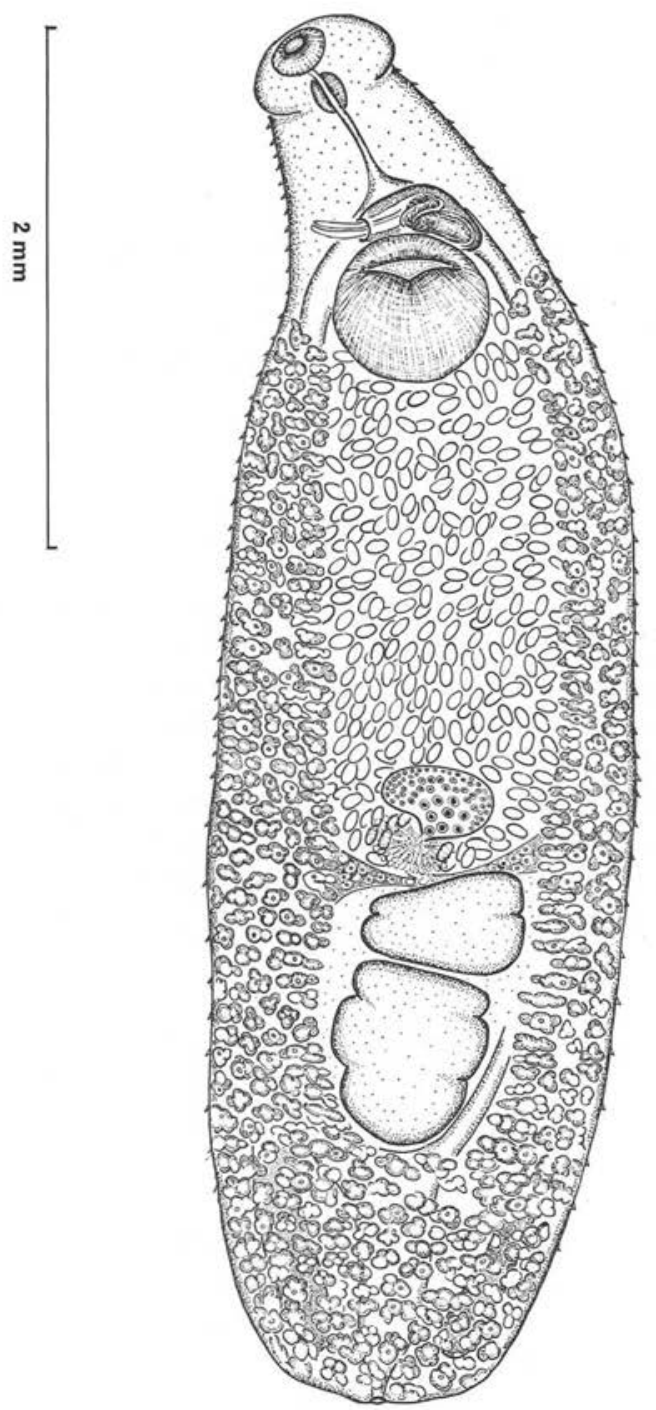

Fig. 4. - Echinostoma togoensis n. sp. Adulte, morphologie générale (vue ventrale). 
ferment des granules très réfringents. La vessie est bilobée ; chaque tronc collecteur s'abouche dans un de ses lobes.

La topographie des éléments sensoriels de la cercaire, en cours d'étude, sera publiée dans un travail synthétique sur la valeur systématique de la chétotaxie chez le genre Echinostoma.

\section{6) Métacercaire (fig. 3b)}

Le kyste métacercarien est phérique. Il mesure 140-170 $\mu \mathrm{m}$ (160) de diamètre. Il est constitué de deux enveloppes d'inégale épaisseur, une enveloppe externe épaisse et une enveloppe interne mince. La métacercaire est repliée à l'intérieur du kyste. Les ventouses, le pharynx et les troncs collecteurs primaires avec leurs granulations sont bien identifiables.

\section{7) Adulte (fig. 4)}

FORME DU CORPS

Corps allongé, à bords latéraux parallèles, présentant un rétrécissement marqué dans la région préacétabulaire. Sur coupes transversales, la section du corps est de forme ovale. Le tégument est recouvert d'épines qui se distribuent principalement sur la face ventrale du corps ; dans sa partie préacétabulaire, quelques épines sont également visibles latéralement et dorsalement. La spinulation devient moins dense dans la région postovarienne et disparaît complètement en arrière du testicule postérieur. Le collier cephalique est armé de 37 épines. La ventouse orale, de petite taille, s'ouvre en position terminale ventrale sur le collier cephalique. L'acetabulum puissant, de forme circulaire, est situé dans le quart antérieur du corps.

\section{Dimensions}

Les dimensions sont celles de 30 adultes de 2 mois obtenus expérimentalement chez la Souris. Les mensurations, obtenues à partir de matériel monté entre lame et lamelle, sont données en $\mu \mathrm{m}$ (les chiffres entre parenthèses représentent les moyennes). longueur du corps

2 590-5 520 (4 580)

largeur du corps

$1200-1550$ (1 375)

ventouse orale

$190-240(210) \times 160-200(185)$

ventouse ventrale

$560-670(615) \times 480-630(555)$

pharynx

$90-170(130) \times 110-200(160)$

longueur de l'oesophage

testicule antérieur

$170-270(190)$

testicule postérieur

$380-560(470) \times 460-590(500)$

$400-700(540) \times 380-550(470)$

ovaire

$150-260(180) \times 280-380(330)$

distance entre l'ovaire et la ventouse ventrale : 690-1 200 (870)

distance entre le testicule postérieur et

l'extrémité postérieure du corps

: 970-1 360 (1 200)

$œ u f s$

$115-130(120) \times 60-70(65)$ 
APPAREIL DIGESTIF

Il comprend un prépharynx, court et étroit, un pharynx, un oesophage étroit et deux caecums intestinaux s'étendant parallèlement aux bords du corps jusqu'à son extrémité postérieure. La bifurcation intestinale est anteacetabulaire.

Appareil génital Male

Les deux testicules, disposés l'un derrière l'autre, sont contenus en gros dans le $3^{\mathrm{e}}$ quart du corps. Leur bord, légèrement invaginé par endroits, leur confère un aspect lobulé.

La poche du cirre, piriforme, épouse le bord antérieur de l'acetabulum. Sa partie postérieure est occupée par une vésicule séminale contournée à laquelle fait suite un cirre musculeux, inerme.

Appareil génital Femelle

L'ovaire, de forme ovoïde, est situé immédiatement en avant des testicules.

Les glandes vitellogènes se distribuent selon deux champs latéraux nettement séparés, sauf dans la région post-testiculaire où ils viennent en contact ventralement. Leur limite antérieure se situe dans la région médio-acétabulaire. Deux vitelloductes transverses, bien individualisés, confluent dans un réservoir vitellin situé au niveau du bord antérieur du testicule antérieur. Entre l'ovaire et le testicule antérieur se développe le complexe de la glande de Mehlis, bien individualisé. Un canal de Laurer s'ouvre dorsalement en position médiane, au niveau du bord postérieur de l'ovaire. Les anses utérines occupent tout l'espace corporel délimité par les vitelloductes transverses, les champs latéraux des vitellogènes et l'acetabulum. L'extrémité de l'utérus n'est pas différenciée en métraterme.

Le pore génital s'ouvre contre le bord antérieur de l'acetabulum, dans la région médiane.

Les œufs, extrêmement nombreux, à coque operculée, sont fortement colorés en brun à maturité.

\section{Recherche expérimentale des hôtes}

1) Transmission au Mollusgue

Les récoltes nous ayant désigné comme hôte naturel Biomphalaria pfeifferi, nous avons testé l'infectivité des miracidiums à l'égard de 3 souches de Biomphalaria d'origine géographique différente : Biomphalaria pfeifferi (souche togolaise), Biomphalaria glabrata (souche guadeloupéenne) et Biomphalaria glabrata (souche albinos brésilienne). Les trois souches peuvent être parasitées par les larves d'Echinostoma togoensis, mais avec un taux de réussite très différent. Chez les deux souches de Biomphalaria glabrata, seuls les très jeunes individus (taille inférieure à $3 \mathrm{~mm}$ ) 
s'infestent normalement avec une réussite variant de 80 à $90 \%$. Les individus de taille comprise entre 4 et $8 \mathrm{~mm}$ s'infestent très difficilement (taux d'infestation compris entre 10 et $15 \%$ ). Au-dessus de $10 \mathrm{~mm}$, aucune infestation n'est possible quelle que soit la dose de stades infestants utilisée. La souche de Biomphalaria pfeifferi d'origine togolaise est réceptive à $100 \%$ même lorsque les infestations sont réalisées avec des individus de taille voisine de $10 \mathrm{~mm}$.

L'infestation du Mollusque est réalisée 10 minutes après l'introduction des miracidiums dans les récipients d'expérimentation. Les coupes histologiques faites 5 heures après l'infestation permettent de recenser les miracidiums dans les couches musculaires sous-tégumentaires de la zone céphalo-pédieuse avec une localisation plus fréquente dans le pied. Au cours de la migration vers le ventricule, les larves empruntent soit les espaces lacuneux, soit les voies sanguines efférentes (artère rectale, aorte antérieure). Les premières larves arrivent dans le ventricule environ 24 heures après l'infestation.

2) Transmission au DeUxiÈme hote intermédiaire

Nous avons réussi les infestations avec les cercaires des espèces suivantes :

- des têtards de Bufo regularis, Hylarana galamensis et Conrana derooi chez lesquels les métacercaires se retrouvent au niveau du tissu rénal;

— des Mollusques Pulmonés d'eau douce : Biomphalaria pfeifferi, Biomphalaria glabrata (souches guadeloupéenne et brésilienne), Bulinus globosus et Bulinus forskalii : chez ces Mollusques, la cercaire s'enkyste dans la cavité péricardique et la portion sacculaire du rein, où les métacercaires forment parfois des amas de plusieurs centaines de kystes.

La migration de la cercaire vers la cavité péricardique emprunte le canal renopéricardique.

3) Transmission au Vertébré

Nous avons tenté l'infestation avec la métacercaire d'Echinosotoma togoensis des Vertébrés suivants : la Souris blanche de laboratoire de souche SWISS, le Rat blanc de race WISTAR, le Rat noir (Rattus rattus), le Canari, le Canard domestique et le Poussin domestique.

Seules les expériences réalisées avec la Souris ont été positives. Les Digènes adultes se localisent chez cet hôte dans la portion distale du jéjunum, où ils atteignent leur maturité sexuelle en 8 jours.

\section{Modalités du cycle dans la nature}

Les données sur les modalités du cycle dans la nature sont encore très fragmentaires. Nous avons recensé le parasite au stade rédie chez Biomphalaria pfeifferi et au stade métacercaire chez Biomphalaria pfeifferi et Bulinus globosus.

L'hôte définitif de ce Digène dans la nature reste à découvrir. 


\section{Appartenance systématique}

Il semble que trois espèces seulement du genre Echinostoma Rudolphi, 1809 sensu stricto aient été signalées à ce jour sur le continent africain et à Madagascar : E. revolutum (Froelich 1802), signalée par Looss (1899) en Égypte ;

$E$. liei, décrite en même temps que son cycle par Jeyarasasingam et al. (1972) en Égypte ;

E. caproni, décrite par Richard (1964) à partir de matériel d'origine malgache et dont le cycle a été récemment élucidé par Richard et Brygoo (1978).

Un certain nombre de caractères à la fois morphologiques et biologiques permettent d'individualiser l'espèce que nous venons de décrire par rapport aux trois espèces citées.

Nos individus montrent peu de parenté avec E. revolutum.

Morphologiquement, ils s'en différencient au niveau de la cercaire par l'existence d'un équipement natatoire plus complexe (E. revolutum ne possède qu'une seule membrane ondulante subterminale dorsale).

Au plan biologique, les différences entre les deux espèces sont marquées : E. revolutum se présente comme une espèce euryxène pouvant évoluer au stade rédie et au stade adulte chez un spectre d'hôtes très large, à la différence de ce que nous avons signalé pour notre espèce. Il semble toutefois que $E$. vevolutum n'ait pas été recensée chez des Pulmonés du genre Biomphalaria, caractère qui l'éloignerait encore de notre espèce.

Notre matériel se différencie de $E$. liei par des caractères à la fois morphologiques et biologiques.

Il s'en éloigne morphologiquement au stade adulte par :

- des dimensions corporelles moins importantes $(4,5 \mathrm{~mm}$ de longueur moyenne contre 6,8 pour E. (iei);

- une position nettement postérieure des glandes sexuelles ;

- l'absence d'espace libre entre le testicule postérieur et la masse des vitellogènes postérieurs.

Les divergences d'ordre biologique sont :

- E. liei offre au stade adulte des potentialités évolutives larges : il peut en effet évoluer chez le Hamster, le Rat noir, le Rat de laboratoire, la Crocidure d'Olivieri, le Poussin domestique, le Canard domestique et le Pinson.

- E. liei réalise avec Biomphalaria glabrata un couple hôte-parasite compatible, contrairement à notre espèce.

Nos individus ne peuvent guère se différencier de E. caproni sur la base de critères morphologiques, au demeurant peu constants.

Les divergences biologiques, par contre, permettent de séparer sans aucune réserve les deux espèces. Ces divergences ont été mises en évidence à partir d'une étude comparative du cycle biologique des deux Digènes entretenus simultanément au laboratoire. 
- E. caproni évolue bien chez les souches brésilienne et guadeloupéenne de B. glabrata, avec des taux de réussite variant entre 50 et $100 \%$ selon la taille des Mollusques. E. togoensis par contre se comporte comme une espèce très peu compatible avec des deux mêmes souches de Planorbes : seuls les Mollusques de taille inférieure à $3 \mathrm{~mm}$ s'infestent.

- E. caproni se développe préférentiellement, au stade sporocyste, dans l'aorte du Mollusque. En ce qui concerne E. togoensis, son microbiotope d'élection est le ventricule, sa localisation dans l'aorte ne s'observe qu'au cours des hyperinfestations.

- E. caproni, parasite naturel d'Oiseaux, évolue dans les conditions de laboratoire à la fois chez des Oiseaux (Poussin, Canard) et des Rongeurs (Souris et Rat blanc). Il semble par contre que $E$. togoensis soit un parasite naturel de Rongeurs, car toutes les tentatives d'infestation d'Oiseaux (Poussin, Canard, Canari) se sont soldées par un échec. Ce Digène a été obtenu expérimentalement exclusivement chez la Souris blanche.

\section{Conclusion}

Le cycle évolutif de $E$. togoensis s'inscrit sans aucune réserve dans le cadre des processus de transmission connus chez le genre Echinostoma ;

- le premier hôte intermédiaire est un Mollusque Pulmoné chez lequel le parasite évolue préférentiellement dans la glande génitale ;

- le deuxième hôte intermédiaire est représenté par des Mollusques Pulmonés de la même espèce ou d'espèces différentes et par des têtards d'Amphibiens.

Une étude préliminaire du développement intravectoriel de E. togoensis a permis de montrer que la multiplication des rédies du parasite s'accompagne systématiquement d'une stérilisation totale du Mollusque. De plus, E. togoensis se montre très pathogène pour son hôte dans les situations d'hyperinfestation. Des recherches sont actuellement poursuivies en laboratoire pour tenter d'apprécier l'importance de ces deux effets à l'échelle des populations de Mollusques, et leur éventuelle utilisation dans la lutte contre les vecteurs africains de S. mansoni.

Remerciements. Nous remercions Madame Brigitte Labrousse pour son précieux concours technique tout au long de ce travail.

Cette recherche a reçu le support financier du Programme Spécial PNUD Banque Mondiale - OMS de Recherche et de Formation concernant les Maladies Tropicales et du C.N.R.S. (ERA 915).

\section{BIBLIOGRAPHIE}

Looss A. : Weitere Beiträge zur Kenntnis der Trematoden — Fauna Aegyptens, zugleich Versuch einer natürlichen Gleiderung des Genus Distomum Retzius. Zoologisches Jahrbuch, Iena Abteilung für Systematische. 1899, I2, 521-784.

Richard J., BRygoo E. R. : Cycle évolutif du Trématode Echinostoma caproni Richard, 1964 (Echinostomatoidea). Ann. Paratisol. Hum. Comp., I978, 53, 265-275.

Jeyarasasingam U., Heyneman D., Hok-Kan Lim, Noshy Mansour : Life cycle of a new echinostome from Egypt, Echinostoma liei sp. nov. (Trematoda: Echinostomatidae). Parasitology, 1972, 65, 203-222.

RICHARD J. : Trematodes d'Oiseaux de Madagascar (note III). Espèces de la famille des Echinostomatidae Poche, 1926. Ann. Parasitol., Hum. Comp., 1964, 34, 607-620. 